\title{
En Chine, le christianisme
}

À propos de :

COMBY Jean, éd., Diffusion et acculturation du christianisme $\left(\mathrm{XIX}^{\mathrm{e}}-\mathrm{XX}^{\mathrm{e}}\right.$ siècles). Vingt-cinq ans de recherches missiologiques. Paris, Éditions Karthala, coll. " Mémoire d'Églises ", 2005, 690 p.

MofFETT Samuel Hugh, A History of Christianity in Asia, 1500-1900. Vol. II, Maryknoll (New York), Orbis Books, coll. « American Society of Missiology Series », 36, 2005, XXVI + 742 p.

OVERMYeR Daniel L., éd., Religion in China Today. Cambridge, Cambridge University Press, coll. « The China Quaterly Special Issues », New Series, 3, 2003, VIII $+235 \mathrm{p}$.

SCHEUER Jacques \& SERVAIS Paul, éds., Passeurs de religions. Entre Orient et Occident. Louvain-la-Neuve, Bruylant-Academia, coll. « Rencontres Orient-Occident », 6, 2004, 428 p.

Françoise Aubin

\section{(2) OpenEdition}

Édition électronique

URL : http://journals.openedition.org/assr/3829

DOI : $10.4000 /$ assr.3829

ISSN : $1777-5825$

Éditeur

Éditions de l'EHESS

Édition imprimée

Date de publication : 1 décembre 2006

Pagination : 75-92

ISBN : 2-7132-2124-2

ISSN : 0335-5985

Référence électronique

Françoise Aubin, «En Chine, le christianisme », Archives de sciences sociales des religions [En ligne], 136 | octobre - décembre 2006, mis en ligne le 07 février 2007, consulté le 19 avril 2019. URL : http:// journals.openedition.org/assr/3829; DOI : 10.4000/assr.3829 


\section{Françoise Aubin}

\section{En Chine, le christianisme}

\section{Généralités}

À propos de :

Comby Jean, éd., Diffusion et acculturation du christianisme (XIX ${ }^{e}$ $X X^{e}$ siècles). Vingt-cinq ans de recherches missiologiques. Paris, Éditions Karthala, coll. " Mémoire d'Églises », 2005, 690 p.

MofFetT Samuel Hugh, A History of Christianity in Asia, 1500-1900. Vol. II, Maryknoll (New York), Orbis Books, coll. «American Society of Missiology Series ", 36, 2005, XXVI + 742 p.

Overmyer Daniel L., éd., Religion in China Today. Cambridge, Cambridge University Press, coll. "The China Quaterly Special Issues ", New Series, 3, 2003, VIII + 235 p.

Scheuer Jacques \& Servais Paul, éds., Passeurs de religions. Entre Orient et Occident. Louvain-la-Neuve, Bruylant-Academia, coll. «Rencontres Orient-Occident », 6, 2004, 428 p.

Les colloques de Louvain-la-Neuve (Belgique) évoquent, les uns après les autres, différents aspects de la rencontre ou de la confrontation, selon les cas, entre l'Orient et l'Occident, la part la plus belle étant faite à la Chine : ainsi ce fut, en 1998, autour de La mort et l'au-delà (volume publié en 1999) ; en 1999 autour de Individu et communauté (2000) ; en 2001 autour de Droits humains et valeurs asiatiques (2002).

Passeurs de religions envisage le processus des échanges religieux à travers l'action de quelques personnalités saillantes. Un vieil exemple sert à démontrer que la Chine a été terre de passage, spécialement à l'époque Tang: c'est au $\mathrm{VII}^{\mathrm{e}}$ siècle que le célèbre moine-pèlerin chinois Xuanzang devint, à son retour d'un séjour de quelque seize ans en Inde, le transmetteur de l'école Yogâcâra (Faxiang en chinois). Disparue en Chine quelques décennies après la mort de Xuanzang, ladite école s'implanta très tôt au Japon, où, sous le nom d'école Hossô (ou Yuishiki), elle s'est perpétuée jusqu'à nos jours (Thierry Marrès, p. 101-111). Quant à la bouddhisation des rituels de l'école taö̈ste Lingbao, elle se produisit sous l'action de la famille Ge, entre le moment de la naissance des grands courants bouddhiques au IV ${ }^{\mathrm{e}}$ siècle et le début des persécutions antibouddhiques du v viècle (Pierre-Henry De Bruyn, p. 223-241). 
Passons aux jésuites des XVII ${ }^{\mathrm{e}}$ XVIII ${ }^{\mathrm{e}}$ siècles : le Français Joachim Bouvet (16561730, en Chine à partir de 1688) remplit de multiples rôles auprès de l'empereur Qing Kangxi (r. 1662-1723) - mathématicien, philologue, cartographe, philologue, etc. - mais la création attachée à son nom est le figurisme chinois qui interprète les caractères de l'écriture chinoise dans un sens théologique et qui fusionne les classiques chinois, notamment le Classique des Mutations (le yijing), avec les récits bibliques (par une grande spécialiste de la question, Claudia von Collani, p. 113-137). L'irremplaçable Handbook of Christianity in China, I, 635-1800 (Leiden, Brill, 2001) a accoutumé les historiens des missions et du christianisme en Chine à aménager leurs matériaux selon des problématiques originales; ici, son inspirateur et rédacteur, Nicolas Standaert, un jésuite lui aussi, offre au même public, avec "Le cas de la Chine au XVII e siècle " (p. 2570), une réflexion féconde sur l'anthropologie de l'analyse du témoignage missionnaire, afin de dégager une méthodologie bonne pour toute étude de l'altérité. L'intérêt de choisir les missions en Chine au XVII siècle vient, premièrement, de la dimension limitée du groupe étudié et du segment de la population autochtone touchée; deuxièmement, du niveau à peu près semblable des cultures impliquées, la chinoise et l'européenne disposant, l'une et l'autre, de l'imprimerie et de l'éducation ; troisièmement, de la quasi absence d'interventions extérieures et de la nécessité pour les Européens d'adopter la culture locale. Le premier cadre d'analyse choisi par les missiologues des années soixante (généralement membres de la congrégation missionnaire qu'ils étudiaient) a été celui de " la transmission " aboutissant à «la conversion » du milieu récepteur, une conception basée sur la distinction aristotélicienne et thomiste du sujet (le transmetteur et le récepteur du message) et de l'objet (c'est-à-dire le message lui-même, censé avoir été immuable). Le deuxième cadre d'analyse, apparu dans les années soixante et soixante-dix, est celui de "la réception ", adopté par les sinologues qui, étudiant les réactions des acteurs chinois eux-mêmes, ont conclu, en général, à l'impossibilité d'une intercommunication correcte. Puis ce fut, sous l'influence des études post-coloniales et culturelles, la théorie de " l'invention » qui a guidé les analyses, en liaison avec les concepts de construction et de déconstruction ; mais l'observateur se signale, au $\mathrm{Xx}^{\mathrm{e}}$ ou $\mathrm{XxI}^{\mathrm{e}}$ siècle, comme le constructeur de la réalité du $\mathrm{XVII}^{\mathrm{e}}$ siècle. Le cadre d'interrogation que propose l'auteur, comme étant le mieux adapté, est, finalement, celui de "l'interaction " et de "la communication ", appuyé sur le concept d'altérité tel que développé, par exemple, par Buber et Levinas, et qui instaure récepteur et émetteur du message comme partenaires dans un dialogue.

Les «passeurs de religion" de la fin du $\mathrm{XIX}^{\mathrm{e}}$ et du $\mathrm{XX}^{\mathrm{e}}$ siècle sont d'abord des Occidentaux qui ont su acquérir une perception particulièrement fine du savoir et de la sensibilité des pays dans lesquels ils ont évolué. Malheureusement, les notices qui leur sont ici consacrées, pour intéressantes et richement documentées qu'elles soient, ont parfois tendance à flirter avec l'hagiographie. Ainsi en 
est-il de la biobibliographie d'Alexandra David-Néel (1868-1969) par une de ses thuriféraires, Joëlle Désiré-Marchand (p. 145-167), qui jamais n'évoque les doutes, bien argumentés, que certains exploits de l'exploratrice ont soulevés chez des témoins de l'époque et des analystes postérieurs. Les autres passeurs sont le jésuite Alfred Bonningue (1908-1997), missionnaire en Chine et en Thaïlande (par une historienne des missions en Chine, Martine Raibaud, p. 211-219); l'abbé Jules Monchanin (1895-1957), prêtre du diocèse de Lyon, qui participa au mouvement de renouveau théologique et liturgique préparant Vatican II et fonda un ashram au Tamil Nadu (par Françoise Jacquin, qui oublie de donner le prénom de son personnage, p. 179-191) ; Jean Herbert (1897-1980), un initiateur de la connaissance de la spiritualité hindoue contemporaine dans le grand public français (par Paul Servais, un des organisateurs du colloque, p. 193-203); Lanza del Vasto (1901-1981), poète et philosophe, défenseur en France d'un pacifisme à la Gandhi (par Bernard Stevens, p. 205-209). Et tout récemment, l'apostolat jésuite à Taiwan a, entre 1951 et 1975, œuvré à appuyer les objectifs éthiques de la politique nationaliste, ainsi dans son enseignement scolaire, et à s'inculturer (par Chen Tsung-ming, p. 373-401).

Un aspect du transfert du message chrétien en Extrême-Orient est l'usage que des mouvements autochtones ont pu en faire : une analyse de l'univers religieux des Taiping en Chine, entre 1851 et 1864 , met de la sorte en lumière ce que l'auteur, Jean-Guy Daigle (p. 243-270), considère comme l'expérience de sinisation la plus poussée du christianisme. Ce peut être aussi la naissance d'un mouvement réagissant contre l'apostolat missionnaire occidental et ses maladresses : en Corée, Philippe Thiébault (p.271-313) mène une enquête fouillée sur le choc qu'a représenté la rencontre du catholicisme pour le Tonghak (littér. "Étude orientale »), une voie religieuse coréenne enracinée dans le confucianisme, que son fondateur, un mystique, Ch'oe Che-U dit Suun, mort martyr à 40 ans (18241864), a tenté de garder en équilibre entre tradition confucéenne et foi catholique. Le caodaïsme vietnamien, religion millénariste instituée formellement en Cochinchine en 1926, fournit, lui, un exemple typique de syncrétisme et de mouvement religieux politisé (Micheline Lessard, p. 359-372). En Chine encore, la "Société de l'Harmonie et la Justice » (Yihetuan), qui a suscité en 1900 le redoutable soulèvement dit "des Boxers", n'aurait pas, selon l'historien taiwanais Chen Fang-chung (p.323-357, avec caractères chinois dans le texte et examen des thèses des historiens de la RPC), été animée avant tout par des sentiments patriotique et anti-impérialistes comme le disent les historiens chinois, mais par la nécessité de lutter contre l'hétérodoxie que représentait l'apostolat missionnaire.

Il faut également se tourner vers l'autre face des contacts interreligieux : la réception en Occident des modes de pensée et des croyances orientales. Lionel Obadia, un spécialiste du bouddhisme tibétain en Occident, propose une réflexion théorique (p. 71-97) sur le bouddhisme et la globalisation, ou la globalisation du bouddhisme devenu au cours du $\mathrm{XX}^{\mathrm{e}}$ siècle une religion planétaire. 
Un des premiers porteurs de la spiritualité orientale en Occident a été le Bengali Rabîndranâth Tagore (1861-1941), le plus ancien prix Nobel non européen en 1913, dont Paul Detienne, un jésuite, loue la diversité des talents (p. 139-147). Interprète du bouddhisme japonais en Occident, le laïc Daisetz Teitaro Suzuki se présente comme un passeur extraordinairement fécond d'un bouddhisme rénové, shin bukkyô en japonais, de tradition Zen/Chan (par le second co-éditeur du volume, Jacques Scheuer, p. 169-177). Le Sôka gakkai, mouvement bouddhiste japonais, fondé en 1930 sur la base du Mahayâna, le Grand Véhicule, s'est internationalisé et rencontre le succès en Occident, grâce à l'impression de sérénité qu'il diffuse, à la simplicité de son rituel, en accord avec une sensibilité nouvelle et à ses activités artistiques (Karel Dobbelaere \& Lilian Voyé, p. 403-412). La Société théosophique, fondée en 1875 par Hélène Blavatsky (1831-1891) et Henry Steel Olcott (1832-1907), a largement exercé sa fascination, notamment sur le mouvement vieux-catholique anglais ou sur des personnalités telles que la pédagogue italienne Maria Montessori (1870-1952), ainsi que le narre Massimo Introvigne dans une contribution trop brève au goût du lecteur (p. 315-321). Deux œuvres picturales de la Taiwanaise Monica Liu, représentant l'une un ascète zen, l'autre une Vierge à l'enfant de style sino-chrétien, ferment cet intéressant Passeurs de religions, dont la lecture est facilitée, tout au long, par une présentation unifiée fort didactique.

Daniel L. Overmyer, éditeur d'un autre ouvrage collectif, Religion in China Today - dont les collaborateurs sont des spécialistes estimés pour les enquêtes sur le terrain qu'ils ont réalisées - est un observateur des mouvements religieux populaires chinois. Aussi la variété des religions dites populaires (malgré le titre qui laisse "religion" au singulier) est, ici, illustrée par plusieurs contributions. À son habitude, Kenneth Dean (p. 32-52) tire de ses enquêtes dans la Chine côtière méridionale une riche problématique, montrant comment le réseau des temples de villages constitue une sorte d'ultime échelon de gouvernement local et comment les rituels participent, quoiqu'on en pense, à la mise en pratique du discours sur la modernité. Un sociologue chinois, Fan Lizhu (p. 53-66), illustre la vigueur des pratiques religieuse paysannes par l'exemple du culte à la déesse Mère des vers à soie perpétué dans un but prophylactique au sein d'un petit village du nord du pays. Selon Liu Tik-sang (p. 67-88), à Hong Kong et Macao, les fêtes des temples sous-tendent activement la vie sociale locale, laquelle évolue dans un monde de pouvoirs surnaturels, malgré une opposition traditionnelle de l'élite cultivée. (N'aurait-il pas valu la peine de signaler que l'anniversaire de la naissance du Bouddha a été déclaré, à Hong Kong, jour férié le 26 mai 1999, d'autant que l'accueil d'une relique sacrée de l'Illuminé, une de ses phalanges, a donné lieu, le 26 mai 2004, à des festivités populaires qui n'avaient rien à envier aux processions en l'honneur de divinités locales ?). À Taiwan, Paul R. Katz (p. 89-106, cf. Arch., 98-43, 1997 \& 116-27, 2001) souligne la force des cultes locaux, particulièrement ceux rendus à Mazu (jadis déesse de la mer) et aux 
Wangye (les Seigneurs royaux, divinités anti peste et anti calamité), et leur imbrication dans la politique de l'île depuis l'abrogation de la loi martiale en 1987 et le développement de la démocratie : ainsi des temples servent de sièges aux réunions pré-électorales, des serments sont pris devant les divinités, notamment par les candidats aux élections, des actes d'accusation sont lus devant elles, par exemple contre des criminels.

Nancy N. Chen, anthropologue de la médecine, livre (p. 199-214) une fine analyse des formes communautaires de charisme curatif par la maitrise du souffle et la méditation dans la Chine post-maoïste : la pratique du qigong est en effet, à partir des années 1980, une réponse à l'effondrement du système de santé et un engagement dans le perfectionnement de soi-même traditionnel ; les exercices, menés en plein air et en groupe sous la direction d'un praticien charismatique, ont un grand effet émotionnel sur les adeptes, en majorité des personnes d'âge mûr ou âgées atteintes de maladies chroniques ou de dépression mentale. Au début des années 1990, le gouvernement sent la nécessité de prendre le contrôle de ces mouvements informels en les démythifiant et en les sécularisant, aussi lance-t-il des campagnes scientifiques afin de séparer les véritables guérisseurs des imposteurs. Les maîtres du qigong recentrent alors leurs empires loin de Pékin ou même à l'étranger et développent des réseaux internationaux, d'abord relayés par les Chinois de la diaspora puis par les Occidentaux séduits aussi. Ainsi en est-il pour une secte charismatique, différente des groupes de qigong en ce que la cure bénéfique s'appuie sur une représentation de la Roue du Dharma (falun) et que les adeptes (deux millions selon le gouvernement, cent millions selon l'information interne) sont mobilisés grâce à des stages d'un enseignement totalement gratuit : le falun gong, prohibé en 1999 par le gouvernement comme " culte malfaisant " (xie jiao) et persécuté depuis lors avec des moyens considérables, notamment par des coups de filet contre les adeptes. Ceux-ci continuent néanmoins à prendre par surprise les forces de police en tenant des meetings de protestation place Tian'anmen les jours de grande fête. Au début du Xxi siècle, la persécution s'étend à tous les mouvements charismatiques, celui des chrétiens évangéliques notamment. Un spécialiste du droit chinois, Pitman B. Potter (p. 1131), remarque justement la difficulté qu'éprouve le gouvernement chinois lorsqu'il veut distinguer la croyance religieuse autorisée et la pratique superstitieuse interdite, afin de maintenir droite la balance entre une tolérance justifiant sa légitimité et le contrôle politique le protégeant des changements socio-économiques.

La présentation des cinq religions, admises officiellement en une forme ecclésiale agréée, renforce l'impression de revivification du religieux dans l'ère postmaoïste que la description de cultes populaires nous avait apportée. Un taoïsant de Hong Kong, Lai Chi-Tim (p. 107-121), dépeint le retour des activités liturgiques taoïstes dans les temples reconstruits, la reprise des ordinations solennelles des prêtres, les Maîtres (daoshi), au monastère " du Nuage blanc » (le Baiyuan guan) de la secte du Quanzhen et dans les salles du Tianshi de la secte du Zhengyi, 
ainsi que la vivacité des rites locaux sous la conduite de Maîtres, mariés et vivant chez eux hors des monastères (les sanju daoshi); l'auteur ne cache cependant pas les dangers qui menacent encore l'avenir du taoïsme. (Peut-être aurait-il fallu ajouter que le Zhengyi, originellement nom de taoïsme du mont Longhu, est maintenant étendu aux taoïstes du mont Mao - école Shangqing, à ceux du mont Gezao - école Lingbao, enfin à tous ceux qui n'appartiennent pas à un monastère particulier, une simplification qui aide l'État à surveiller bon nombre de pratiques poursuivies hors monastères). Le bouddhologue Raoul Birnbaum (p. 122-144), s'interrogeant sur la consistance de la vie monastique bouddhique dans la Chine à l'aube du XXI ${ }^{e}$ siècle remarque que les questions qui se posent à ce sujet de nos jours, concernant la formation et la direction du personnel monastique ainsi que la gestion économique des monastères, sont héritées de celles qu'ont entraînées les innovations de la fin des Qing et de l'époque républicaine, de la fin du XIX ${ }^{\mathrm{e}}$ au milieu du $\mathrm{XX}^{\mathrm{e}}$ siècle. (Ajoutons à la bibliographie de l'auteur, concernant les ouvertures du bouddhisme dans le siècle qui a précédé le communisme, Gabrièle Goldfuss, Vers un bouddhisme du Xxe siècle. Yang Wenhui (1837-1911), Paris, Institut des Hautes Études chinoises, 2001 ; et, à propos des liens possibles entre les bouddhismes chinois et tibétain, Ester Bianchi, The Iron Statue Monastery. "Tiexiangshi". A Buddhist Nunnery of Tibetan Tradition in Contemporary China, Florence, Leo S. Olschki Editore, 2001). L'absence du bouddisme tibétain comme religion indépendante est un choix de l'éditeur qui n'a pas voulu paraître cautionner l'idée que le Tibet pourrait faire partie intégrante de la Chine; le bouddhisme Jaune aurait cependant pu être évoqué en liaison avec les Mongols de Mongolie-Intérieure et de l'Ouest de la Chine.

Dru C. Gladney (p. 145-161), grâce à qui l'islam sinophone est maintenant reconnu par les sinologues comme une religion chinoise à part entière ( $c f$. Arch., 102-83, 1998), a choisi de traiter ici l'ensemble des peuples musulmans de la République populaire chinoise depuis les événements du 11 septembre et la lutte antiterroriste afin de dessiner le large éventail des solutions choisies par les uns et les autres : les Hui sinophones sont les plus intégrés de tous les peuples musulmans de la RPC et cela depuis plusieurs siècles déjà ; les Uyghur (Ouïgours), turcophones, sont les plus résistants à la sinisation et les seuls à présenter des revendications d'autonomie (leur diaspora ne compte pas moins de vingt-cinq organisations internationales et sites web réclamant l'indépendance du « Turkestan oriental ", depuis des bases installées à Istanbul, Ankara, Almaty, Amsterdam, Munich, Melbourne et Washington, p. 151-152). Il est des peuples turcophones (Uzbek, Kazakh, Kyrghyz) ou indo-européens (Tajik) qui maintiennent solidement leur langue et leur culture, il en est d'autres qui s'accommodent de la sinisation (les mongolophones Dongxiang et Bao'an). Et à l'intérieur de chaque bloc culturel, les options scripturales (le fondamentalisme textuel) ou mystiques (le soufisme individuel), traditionnelles (le culte en mosquée) ou associatives et réformatrices (les confréries soufies, dites menhuan en chinois), sont multiples, en particulier 
chez lesdits Hui. Ainsi se trouve battu en brèche le préjugé accrédité par les premiers historiens de l'islam en Chine (les missionnaires protestants puis Raphael Israeli notamment) selon lesquels l'islam ne pouvait être que fauteur de troubles antiHan et instigateur de sécession. L'on regrettera toutefois que l'auteur oublie parfois en route de prendre par la main son lecteur non spécialiste : il omet, par exemple, de préciser que Hu Dengzhou (p. 160) est le premier pédagogue connu de l'islam sinophone et qu'il a vécu au Xvi siècle, que le penseur Liu Zhi n'est pas non plus un héros moderne mais que ses dates sont à cheval sur les XVII e$^{-}$ $\mathrm{XVIII} \mathrm{e}^{\mathrm{e}}$ siècles, que le gedimu, fréquemment mentionné, est le système traditionnel du culte géré par la mosquée, par opposition à la confrérie soufie (le terme chinois ge-di-mu rendant l'arabe qadim, "ancien »).

Spécialiste du catholicisme en RPC, Richard Madsen (p. 162-181), dégage habilement les couches entremêlées de la communauté catholique de Chine et l'orientation différente que chacune a suivie. À la base, l'on trouve la masse des paysans, regroupés souvent dans de petits villages fiers d'être demeurés presque totalement catholiques; respectueux des traditions et de l'autorité du prêtre, ils attendent des rites la sanctification personnelle. Par contre, pour les nouveaux prêtres, les sacrements représentent avant tout de puissants symboles de la foi, et ils se sentent tiraillés entre les leçons de Vatican II, difficiles à intérioriser, et le style de vie et de croyances que leur imposent les paysans. Quant aux jeunes qui quittent leur campagne pour la ville, ils y perdent souvent la foi. Les séminaristes ou les tout jeunes prêtres renvoyés à la campagne ont tendance, eux, à modeler leur conduite sur celle des cadres ruraux, tandis que se pose, d'une manière aiguë, le problème de l'adaptation des pratiques pastorales à un public de citadins touchés par le mercantilisme. Les relations avec le gouvernement sont très politisées et le Vatican est engagé avec le Parti communiste chinois dans un jeu incertain de coopération et de conflit pour la mainmise sur le contrôle de l'Église catholique. La présentation du protestantisme par une autorité en la matière, Daniel H. Bays (p. 183-198), cherche à répondre aux interrogations les plus pressantes. Quelle est l'importance numérique du groupe ? Les statistiques vont d'un chiffre officiel de quinze millions (en 2000) à l'évaluation de cinquante millions lancée par des observateurs spécialisés (pour les catholiques, on parle de dix à douze millions en 2001, avance R. Madsen, p. 163). Il ne fait pas de doute que les forces du protestantisme qui, avant l'arrivée du communisme, étaient très inférieures à celles du catholicisme (on parlait alors de trois millions de catholiques et sept cent mille à un million de protestants), l'emportent maintenant en nombre. Les croyances, les rites et les organisations ecclésiales sont marqués, à la fois, par l'héritage des dénominations missionnaires et par les prises de position des mouvements évangéliques autochtones fondés dans la première moitié du XXe siècle; il ne faut pas oublier non plus qu'une grande partie des nouveaux convertis a été formée par la culture religieuse populaire. Il en est résulté une évolution vers un sectarisme rural que le protestantisme orthodoxe est en droit 
de qualifier d'hérétique, avec des leaders charismatiques se prenant pour le Christ ou pour Dieu. Cependant des intellectuels, généralement convertis eux-mêmes, avancent que certaines valeurs du christianisme pourraient participer au mouvement de modernisation du pays.

Un ouvrage du même nom était déjà paru en 1989 : Religion in China Today. Policy \& Practice de Donald E. MacInnis (Maryknoll, Orbis Books), un recueil de cent trente-sept documents révélant la politique religieuse entre 1979 et 1988 et, par des interviews et des récits autochtones, la pratique des cinq religions officielles ainsi que de l'orthodoxie russe, du judaïsme, du confucianisme, du marxisme comme foi personnelle, des cultes populaires enfin. Si le champ du présent ouvrage est plus restreint, les religions populaires y reçoivent une attention plus poussée et chaque religion y fait l'objet d'une synthèse qui, riche en problématiques, constitue chaque fois une heureuse introduction au sujet.

Samuel H. Moffett, fils d'un missionnaire presbytérien en Corée, a eu, au début des années 1990, le courage de quitter sa spécialité, le christianisme en Corée, pour se lancer dans une vaste fresque du christianisme en Asie depuis ses origines. Le premier tome de A History of Christianity in Asia (San Francisco, Harper Collins, 1992 ; $2^{\mathrm{e}}$ éd. revue, Maryknoll, N.Y., Orbis Books, 1998), qui prenait l'histoire de l'Église d'Orient de ses débuts jusqu'à sa fin en l'an 1500, a été salué par la critique comme une synthèse remarquable, unissant des narrations et des discussions théologiques facilement accessibles au non spécialiste. Les compliments peuvent être, sans effort, reportés sur le second tome qui, lui, couvre l'histoire missionnaire et la constitution des communautés chrétiennes autochtones : rien d'équivalent n'avait jamais été tenté et il est à prévoir qu'une telle somme restera longtemps l'ouvrage de référence fondamental.

Une première partie, en neuf chapitres, couvre les missions catholiques jusqu'au XIX siècle : les Portugais en Inde et en Asie du Sud-Est, les jésuites au Japon et en Chine, les Espagnols aux Philippines, et en outre les églises moyenorientales - nestorienne, jacobite, arménienne -, sous la domination turque et persane aux XVI ${ }^{\mathrm{e}}$ et $\mathrm{XVII}^{\mathrm{e}}$ siècles. Une très brève deuxième partie reprend l'histoire du point de vue protestant aux XVII $-\mathrm{XVIII}^{\mathrm{e}}$ siècles : les Hollandais en Indonésie, à Formose et à Ceylan, les piétistes d'Europe septentrionale en Inde. La grande affaire est la troisième partie, qui occupe les trois quarts du volume : le " grand siècle » des missions, de 1784 à 1860, au dire de l'auteur. Une section 1 est consacrée à la « compétition » entre protestants et catholiques, en Inde, en Chine, en Corée, en Birmanie, à Ceylan, en Asie du Sud-Est, avec un retour sur les nestoriens, les arméniens et les maronites du Moyen-Orient. Malgré la remarquable impartialité de ses fresques, l'auteur ne peut s'empêcher de souligner, pour le catholicisme, les échecs et, pour le protestantisme, les victoires et les essors; la présentation du titre de la section " protestants et catholiques » est déjà le signe d'une légère préférence. Mais on ne saurait lui en faire grief: les histoires générales du christianisme en un ou plusieurs pays d'Asie sont généralement 
marquées par l'appartenance religieuse de leurs auteurs, et souvent beaucoup plus nettement que ne le fait S.H. Moffett. Bien que l'intitulé de cette troisième partie se donne pour limite chronologique 1860, la section 2 qui la subdivise s'étend de 1860 à 1900 (période que j'aurais vue comme le grand moment des missions, plutôt que les huit décennies précédentes), pour dépeindre la " maturité » atteinte par les missions (surtout protestantes) et la montée des églises autochtones.

Le procédé principal, auquel recourt l'auteur, est celui de la narration événementielle, attentive aux détails et à la psychologie des acteurs d'après leurs lettres ; les informations sur les stratégies missionnaires se glissent tout naturellement dans le récit, sans que le lecteur peine pour suivre le fil de la démonstration ni que le plaisir de sa lecture en soit troublé. De fait, ce gros ouvrage se lit presque comme un roman où abondent les héros et les situations tragiques, d'autant que les notes sont reportées à la fin de chaque chapitre pour ne pas entraver le fil du récit. L'information est cependant considérable, renforcée par des tables chronologiques qui ferment les chapitres les plus stratégiques, ainsi les événements du Japon entre 1500 et 1708 (p. 93-96) et le mouvement missionnaire protestant entre 1792 et 1859 sur fond d'histoire de l'Inde et de missions catholiques peu visibles (p. 270-274) et par des cartes géographiques claires à souhait. Parmi les points intéressants soulignés par l'auteur, relevons, en Chine, le problème du développement de l'apostolat féminin en milieu protestant : il note le rôle qu'a joué la China Inland Mission, société missionnaire interdénominationnelle fondée en 1866, dans l'admission de femmes célibataires sur le terrain et dans la reconnaissance des épouses de missionnaires comme collaboratrices de plein droit, ainsi que dans l'autorisation de mariages mixtes sino-britanniques (p. 466-469 \& 477-479), les difficultés surgies en Corée pour les premiers baptêmes de femmes dans les dernières décennies du XIX ${ }^{e}$ siècle (p. 533).

L'apport bibliographique est considérable, occupant soixante-huit pages en une typographie serrée. Au premier coup d'œil, elle semble être uniquement anglophone; mais un examen plus attentif révèle, sauf erreur de ma part, une quarantaine de titres en français (les ouvrages classiques de Jacques Gernet sur Chine et christianisme et de Jean Lacouture sur les jésuites sont présents en traduction anglaise), vingt-cinq en allemand, six en néerlandais, cinq en latin, trois en italien, deux en espagnol, un en portugais, un en coréen, ce qui n'est somme toute pas mal. Si la bibliographie concernant le protestantisme paraît impeccable, le côté catholique de l'histoire est victime de trous surprenants, du moins dans l'aire culturelle dont je peux juger, la Chine : il manque en effet des travaux aussi fondamentaux que ceux de Nicolas Standaert (Yang Tingyun, Confucian and Christian in Late Ming China, sur lequel voir Arch., 67, 1989, p. 175 \& 187-188) et Gianni Criveller (Preaching Christ in Late Ming China, voir Arch., 110-15, 2000) et, in texto, le regard neuf qu'ils permettent de jeter sur le catholicisme autochtone du XVII ${ }^{\mathrm{e}}$ siècle. Les missions, protestantes autant que catholiques, en Mongolie-Intérieure, chez les colons chinois et chez les 
Mongols de l'Ordos (la seule mission catholique chez les Mongols avant 1990 : cf. infra), ont été oubliées, bien qu'une publication de la congrégation catholique d'origine belgo-néerlandaise, CICM dite Scheut, concernée par le sujet, apparaisse dans la bibliographie (Historiography of the Chinese Catholic Church) contenant plusieurs articles sur son histoire en Chine. Un lazariste belge, le P. Vincent Lebbe (1877-1940), dont l'action, certes contestée, en son temps et maintenant encore, pour avoir été exagérément médiatisée, ne peut être ignorée tant elle préfigurait avec panache, dans les années 1920 et 1930, les rapports actuels avec le pouvoir communiste, faits d'égalité statutaire entre Occidentaux et Chinois et d'indépendance des Églises autochtones (sa biographie est donnée justement dans Historiography of the Chinese Catholic Church). Si une réédition revue et complétée de ce splendide ouvrage était prévue, il faudrait demander à l'auteur, outre un léger appoint documentaire, un index moins étique et incomplet que celui, actuel, dans lequel on ne trouve généralement pas ce que l'on y cherche. Quant à la force numérique du christianisme en 2000 (p. 488), une question d'ailleurs ici hors sujet, le chiffre donné pour le catholicisme semble sous-évalué (sept millions) et celui du protestantisme surévalué (soixante et onze millions, sur la foi du World Christian Encyclopedia de 2001).

Diffusion et acculturation du christianisme est un choix de quarante contributions (plus deux articles, en prologue, racontant la naissance du CREDIC et ses méthodes, et, en conclusion, deux sur la controverse autour du terme d'inculturation) extraites des actes des colloques du CREDIC (Centre de recherches et d'échanges sur la diffusion et l'inculturation du christianisme), une association œcuménique d'étude missionnaire lancée à Lyon en 1979, en pleine crise de la décolonisation et de l'indépendance des pays en voie de développement. Les réunions, annuelles, tournent autour d'un thème donné, depuis le premier colloque tenu à Fribourg (Suisse) en 1980 jusqu'au vingt-cinquième, en 2004, à Belley. Plusieurs des recueils qui en ont résulté (et d'où certains des présents articles sont tirés) ont déjà été recensés dans les ASSR : ainsi, au volume 86-14, 1994, sur Des missions aux Églises: naissance et passation des pouvoirs, XVII ${ }^{e}$ XX $X^{e}$ siècles, $\mathrm{X}^{\mathrm{e}}$ colloque du CREDIC, 1989, Bâle ; $\mathrm{n}^{\circ}$ 15, sur Femmes en mission, $\mathrm{XI}^{\mathrm{e}}$ colloque, 1990, Saint-Flour; $\mathrm{n}^{\circ} 16$, sur Sciences de la mission et formation missionnaire au XX ${ }^{e}$ siècle, $\mathrm{XII}^{e}$ colloque, 1991, Vérone ; au volume 126-11, 2004, sur Ecuménisme et pratiques missionnaires, $\mathrm{XXI}^{\mathrm{e}}$ colloque, 2000, Louvain-laNeuve; $\mathrm{n}^{\circ} 25$, sur L'espace missionnaire. Lieu d'innovations et de rencontres interculturelles, XXII ${ }^{e}$ colloque, 2001, Québec.

Huit contributions ont été retenues pour traiter de l'émergence des sciences de la mission : par exemple, du côté catholique, d'abord les réductions jésuites du Paraguay aux XVII ${ }^{e}$-XVIII ${ }^{e}$ siècles, puis, à l'époque moderne, le chanoine Joly depuis Paris, le P. Lebbe en Chine, le P. Charles à Louvain; du côté protestant, le rôle de Maurice Leenhardt en Nouvelle-Calédonie. Pour définir les lieux et pratiques de la mission, encore huit articles sur Ozanam et la Propagation de la 
Foi, sur les Frères de Ploërmel au Sénégal, sur la mission catholique allemande au Cameroun de 1896 à 1916, ou encore sur le sacre des six premiers évêques chinois en 1926 vu par la diplomatie française ; et, pour les missions protestantes, leur action en Polynésie et aux Philippines. Quatre auteurs réfléchissent aux risques de la traduction religieuse et à ses réussites et six se penchent sur les diverses manifestations du laïcat, notamment sur le rôle des femmes en mission. Parmi les quatre articles formant la section "Presse, iconographie ", on remarquera celui de Jean Pirotte sur l'imagerie de dévotion au XIX ${ }^{\mathrm{e}}$ siècle (p. 457-470). Les dix dernières contributions sont consacrées aux mutations de la seconde moitié $\mathrm{du} \mathrm{Xx}^{\mathrm{e}}$ siècle et au déplacement des missions vers les Églises locales, d'une part dans la problématique débattue au sein du catholicisme et du protestantisme, d'autre part, dans la réalité, ainsi au Vietnam ou en Afrique. Cette somme d'un quart de siècle de réflexion missiologique, croisée entre catholiques et protestants, est la conclusion stimulante d'une belle réussite.

\section{Missions en Chine et Églises locales aux temps modernes}

Ducornet Étienne, L'Église et la Chine. Histoire et défis. Paris, Les Éditions du Cerf, coll. "Histoire du christianisme », 2003, 180 p.

EtChegaray Cardinal Roger, Vers les chrétiens en Chine vus par une grenouille $d u$ fond $d u$ puits. Paris, Éditions du Cerf, 2004, 99 p.

Heylen Ann, Chronique du Toumet-Ortos. Looking through the Lens of Joseph Van Oost, Missionary in Inner Mongolia (1915-1921). Leuven, Leuven University Press \& Ferdinand Verbiest Foundation, coll. "Leuven Chinese Studies ", XVI, 2004, 409 p.

LEUNG Beatrice \& CHAN Shun-hing, Changing Church and State Relations in Hong Kong, 1950-2000. Hong Kong University Press, coll. "Hong Kong Culture and Society ", 2003, XVIII + 230 p.

TAVEIRnE Patrick, Han-Mongol Encounters and Missionary Endeavors. A History of Scheut in Ordos (Hetao), 1874-1911. Leuven, Leuven University Press \& Ferdinand Verbiest Foundation, coll. « Leuven Chinese Studies », XV, 2004, 684 p.

Les deux premiers ouvrages de la présente section concernent une société missionnaire catholique, dont il a été question dans les ASSR à plusieurs reprises : la Congrégation belge du Cœur Immaculé de Marie (CICM), dite de Scheut, du nom du faubourg de Bruxelles où elle a été fondée en 1862-65, pour accomplir une œuvre missionnaire en Chine, sur la base d'un recrutement alors majoritairement flamand et néerlandais (cf. Arch., 86-67, 1994, sur son historique ; 11075, 2000, \& 122-28, 2003, sur des volumes collectifs édités à l'Université de Louvain, Leuven, par la société érudite qu'elle patronne maintenant, la Fondation Ferdinand Verbiest). Son apostolat s'est principalement exercé parmi de pauvres paysans chinois émigrés au-delà de la Grande muraille, dans les steppes de la Mongolie méridionale. 
Ainsi, La chronique du Toumet-Ortos éditée par Ann Heylen est un journal. Du côté catholique, deux contributions seulement : le témoignage d'un homme politique converti au catholicisme, John C.H. Wu, par Matthias Christian; d'abord trimestriel puis devenu quotidien au fil du temps, tenu en français, entre janvier 1915 et mai 1921, par un scheutiste flamand, excellent francophone, le P. Joseph Van Oost (1877-1939, arrivé en Chine en 1902), depuis des petits postes répartis parmi la population chinoise, entre les monts Yin-shan au Nord (localement appelés Daqing-shan) et la grande boucle du fleuve Jaune, au sudouest de l'actuelle capitale de la Mongolie-Intérieure, Hohhot (jadis dénommée en mongol Köke-qota, nom dont la prononciation moderne est devenue Hohhot, et dite en chinois avant l'ère communiste Guihuacheng, c'est-à-dire "la Ville ralliée à la civilisation », ou encore Guisui, c'est-à-dire les villes jumelées de Guihua, la ville chinoise, et de Suiyuan, la ville mandchoue - des noms dont Ann Heylen n'a pas bien saisi l'identité géographique ni la différence sémantique). Il est dommage qu'elle n'ait pas donné la moindre carte, tant pour localiser la région concernée dans l'ensemble chinois, que pour aider le lecteur à se retrouver dans la multitude des noms de lieux où se déroulent les événements narrés par Van Oost, d'autant que ce dernier en avait dressé une bonne cartographie (mais sans échelle) dans ses Notes sur le Toemet publiées en 1922. Ann Heylen a choisi de sélectionner (au prix de quelques répétitions), selon sept thèmes dominants, de larges extraits des trois gros cahiers manuscrits, que leur auteur destinait visiblement à une publication dans la Revue de Scheut et dont il n'a publié que quelques morceaux dans d'autres périodiques missionnaires. Le texte écrit de la main du P. Van Oost a été saisi sur ordinateur par le P. Albert Cornelis, CICM (un Wallon né en 1925). A. Heylen y a ajouté, en anglais, son propre commentaire historique, qui réussit le tour de force de ramasser en quelques paragraphes le contenu des travaux historiques fondamentaux traitant des situations et des événements mentionnés dans la chronique originelle (on regrettera qu'elle ait cité en flamand des publications dont il existe une version française bien avérée, telle l'histoire de Scheut par D. Verhelst \& H. Daniels, dont la version flamande originale est de 1991, la version française de 1993 et l'anglaise de 1995).

Les deux premiers chapitres mettent le décor en place : le cadre écologique et socioreligieux, où sont passés en revue les fêtes religieuses populaires, les prières pour la pluie, les rites des cérémonies familiales, le concept de mort. Le ton est enjoué, le récit amusant ou prenant. Comme le souligne Ann Heylen, ce ne sont pas tant les faits qui intéressent, que l'ambiance de l'époque qui les entoure ; et le goût évident de l'auteur pour l'écriture donne une chaleur communicative à ses récits, ici différents de ceux rapportés dans ses Notes sur le Toemet publiées ultérieurement (ce nom de Tümet n'est pas « aussi » le nom d'une tribu mongole, comme le dit la commentatrice, p. 27, n. 1, mais principalement le nom de la tribu qui occupait le territoire avant d'en être chassée par la colonisation chinoise - la photo de femmes assises, p. 67, représente d'ailleurs des Mongoles et non des Chinoises, comme le contexte pourrait le laisser croire; quelques autres 
petites bévues dans le domaine mongol peuvent être relevées de-ci de-là, ainsi, p. 390, le nom d'un auteur mongol dit «Te, Mole» et qui doit être compris, non pas « Monsieur Te, prénommé Mole », mais « Temür », un nom personnel mongol très répandu).

Le troisième chapitre consacré au travail missionnaire sur le terrain décevra sans doute le missiologue amateur, bien qu'il ne soit pas dépourvu d'informations factuelles: l'hécatombe que le typhus et la peste bubonique causent dans les rangs des jeunes prêtres européens, l'ordination de prêtres chinois, les écoles catéchuménales et la répétition du catéchisme à l'occasion des grandes fêtes d'obligation, les solennités religieuses rehaussée par une musique cacophonique. Mais, d'une façon générale, les chrétiens chinois sont pratiquement absents de ce recueil de souvenirs. Les quatre chapitres suivants dissèquent les drames de la réalité politique et sociale vus d'en bas. Au premier abord, il est un peu déroutant pour le lecteur de vivre au milieu du banditisme endémique et de ses horreurs (chap. 4) avant d'en comprendre l'ancrage dans la politique nationale (chap. 5), dans les essais avortés de réformes, concernant notamment la culture et le commerce de l'opium (chap. 6) et dans le contrecoup de la Première Guerre mondiale (chap. 7). Mais finalement ce choix chronologique n'est pas plus mauvais qu'un autre et fait ressortir de façon vivante et immédiate l'affreuse insécurité vécue par les agriculteurs chinois de la Mongolie méridionale et la place que l'Église catholique missionnaire essayait de tenir au milieu de la débâcle.

Le P. Van Oost résidait en Mongolie-Intérieure, certes, mais dans une zone de peuplement chinois et non pas mongol. La remarquable somme du P. Taveirne, CICM, Han-Mongol Encounters and Missionary Endeavors, concentrée sur une région proche, l'Ordos à l'intérieur de la grande boucle du fleuve Jaune, traite, elle, des Mongols autant que des Chinois, et de bien d'autres choses encore. Qu'on ne s'y trompe pas : cet ouvrage, qui élève très haut le niveau des études missiologiques, est un modèle du genre ; et il semble qu'il sera difficile désormais de faire de l'histoire missionnaire et chrétienne, en Chine du moins, sans avoir en vue l'ampleur encyclopédique des problématiques qu'il a envisagées. Peutêtre lui reprochera-t-on sa trop grande richesse qui peut en décourager la lecture, et d'avoir voulu unir en un seul gros recueil la matière de plusieurs traités différents. Les destinataires de chacun de ces travaux penseront-ils à consulter un ouvrage au titre aussi peu prometteur ?

Un des volets de Han-Mongol Encounters and Missionary Endeavors est une histoire des rapports des Mongols méridionaux avec la puissance sino-mandchoue, l'avancée de la présence chinoise en terre mongole et une vue d'ensemble de la rébellion des Boxers en 1900. Un autre est un survol d'ensemble de l'histoire des missions en général, et en Chine particulièrement, missions protestantes et orthodoxes incluses. Et, bien sûr, toute l'histoire de la fondation en Belgique de la congrégation de Scheut (CICM) et de son installation en Mongolie-Intérieure, plus précisément en Ordos, dont elle avait reçu la charge en 1864 et où elle a ouvert la seule mission destinée aux Mongols, outre celles visant les colons 
chinois un peu partout en Mongolie-Intérieure. L'historien des missions trouvera là, sur la base des archives de Scheut (citées en note dans leur langue d'origine, français ou flamand), des réponses à bien des questions qu'il peut se poser sur l'origine familiale, la formation scolaire puis théologique du future missionnaire, l'entretien de sa spiritualité une fois sur le terrain, les tensions entre tradition et rénovation de la pratique rituelle, la correspondance avec les donateurs, etc. (cependant, les questions financières ne sont pas abordées en tant que telles). On pourra remarquer, grâce aux tableaux finals, que les grandes figures de la congrégation sont issues de familles nombreuses, ce qui n'est pas le cas pour tout le monde: Mgr A. Bermyn est le septième de dix enfants ; l'infortuné Mgr F. Hamer, qui mourra torturé par les Boxers, est le huitième de neuf enfants ; Mgr O. Hubert, le cinquième de huit, Mgr J. Van Aertselaer, futur supérieur général de la congrégation, le septième de huit (p. 623-628). Il n'est pas jusqu'à l'historien de la Belgique qui ne puisse y glaner, par exemple en trouvant confirmation du prestige du petit séminaire de Hoogstraten, en Belgique septentrionale (p. 634).

Enfin, le présent travail est une perle dans l'histoire missionnaire extrêmeorientale par la place qu'il laisse aux catéchumènes et chrétiens chinois et mongols. Voici un livre qui se préoccupe des jugements autochtones portés sur le christianisme et sur la stratégie d'acquisitions foncières des pères de Scheut en Ordos, un livre décrivant les pratiques pieuses, les fêtes calendaires et les rites de la vie chez les Mongols d'une part, chez les Chinois de l'autre, qui offre, ainsi, une information originale aux ethnologues mongolisants autant que sinologues.

Le petit volume du P. Étienne Ducornet, L'église et la Chine, pourrait passer inaperçu, étouffé par l'ampleur thématique et matérielle de la somme dressée par le P. Taveirne. Ce serait grand dommage, car voici, sous un volume réduit, une vue d'ensemble, agréable et convaincante, des problèmes que rencontre l'implantation du christianisme, et plus particulièrement du catholicisme en Chine (avec quelques aperçus sur le protestantisme). En peu de mots tout est dit du passé, sur la base d'une bonne connaissance de la culture traditionnelle et de l'histoire missionnaire, et surtout du présent, dans des observations nourries par une connaissance réelle du terrain. Ainsi en est-il du thème de la grotte : grottes de Lourdes à l'entrée des églises chinoises répondant aux grottes du bouddhisme et du taoïsme ; ou statues de la Vierge dans le style des Guanyin bouddhiques (p. 111). La description de la situation actuelle s'articule autour de trois défis. Le premier défi est celui de l'inculturation, en quelque sorte " une incarnation culturelle » de la foi : "Et le Verbe s'est fait idéogramme », selon une excellente formule (p. 96) qui peut résumer l'aventure culturelle qu'a représentée l'intégration du christianisme dans la culture chinoise. Puis vient le défi de la communion, " un seul baptême, une seule foi, une seule église ", qui est celui auquel doivent faire face l'Église officielle et l'Église clandestine. Ici, le propos est enrichi de réflexions cueillies sur place. Le troisième défi, qui n'est pas des moindres, est celui de la modernité, et surtout d'une modernité aux accents chinois. La conclusion 
s'intitule joliment «de l'Empire du Milieu au Royaume des Cieux ». En annexe, le message que Jean-Paul II fit parvenir aux participants d'un congrès international « Matteo Ricci : pour un dialogue entre la Chine et l'Occident » en 2001.

L'initiation à la situation du christianisme en Chine populaire peut se poursuivre avec les souvenirs chinois du Cardinal Etchegaray. Le titre même, inspiré par un dicton local, Vers les chrétiens en Chine vus par une grenouille $d u$ fond $d u$ puits, annonce un récit familier, qui éclaire autant sur la personnalité sympathique d'un auteur fier de son origine basque et de son village natal d'Espelette, que sur l'attitude des organismes officiels chinois à l'égard de la religion et de son représentant étranger, " haut fonctionnaire d'une religion occidentale " (p. 71), différente à chacun des voyages du prélat (1980, 1996, 2000, 2003). En annexe, le message de Jean-Paul II donné aussi par le P. Ducornet.

Beatrice Leung (Liang Jiefen en langue standard de Pékin), une catholique spécialiste bien connue des rapports entre la Chine et le Vatican, et Chan Shun-hing (Chen Shenqing en pékinois), un protestant préoccupé par les incidences philosophiques de l'implantation du christianisme en milieu chinois, se sont unis pour réaliser une analyse très fouillée des rapports entre Églises et État à Hong Kong: Changing Church and State Relations in Hong Kong, 1950-2000. Une riche bibliographie en chinois et un glossaire donnant les caractères chinois de tous les noms de personnes et d'institutions garantissent le sérieux de l'entreprise. L'étude, qui commence à l'ère de la colonisation britannique, expose d'abord la coopération des Églises avec le gouvernement en matière d'éducation et de santé ; puis les difficultés de l'ajustement des relations à l'époque transitoire des années 1980 à 1997, et le conflit entre l'aile libérale des Églises et les conservateurs. Depuis la translation de la région de Hong Kong au régime populaire, les relations suivent le modèle théorique dit "contractuel ", dont elles sont une illustration en action. Apport à l'histoire de Hong Kong et à celle du christianisme asiatique, cette étude est également une réflexion sur la théorie des rapports entre État et religion.

\section{La bible et le Christ en Chine}

GálIK Marián, Influence, Translation and Parallels. Selected Studies on the Bible in China. Sankt Augustin, Monumenta Serica Institute, coll. «Collectanea Serica », 2004, 351 p. Introd. par Irene Eber.

Malek Roman, svd, éd., The Chinese Face of Jesus Christ, vol. 1-3. Sankt Augustin, Institut Monumenta Serica \& China-Zentrum, coll. « Monumenta Serica Monograph Series », L/1-3, 2002, 1311 p.

Vu la quantité de travaux qui lui ont été consacrés depuis le XIX siècle, tout spécialement par les milieux missionnaires protestants, l'aventure de la bible en Chine pourrait paraître un thème désormais épuisé. Or il n'en est rien. Le 
P. Malek, svd (congrégation d'origine allemande du Verbe Divin, dite de Steyl), qui dirige à St Augustin près de Bonn l'institut sinologique Monumenta Serica et la revue érudite du même nom, déploie une belle énergie pour revivifier sans cesse le sujet. En témoignent deux publications qu'il a dirigées coup sur coup : Bible in Modern China. The Literary and Intellectual Impact (1999, cf. Arch. 110-59, 2000) et par J.O. Zetsche, The Bible in China. The History of the Union Version (1999, cf. Arch., 114-48, 2001). Ici, il a permis à un spécialiste tchèque de la littérature chinoise et du comparatisme littéraire, Marián Gálik, longtemps empêché par le régime communiste de pouvoir traiter librement de la question, d'exposer sa prodigieuse érudition en matière de traitement, ou de parallèles, de thèmes bibliques dans la littérature chinoise. Influence, Translation and Parallels. Selected Studies on the Bible in China est une collection d'articles séparés, fortement personnels pour beaucoup d'entre eux. L'importance de la bible - étant entendu qu'il s'agit, tout au long du présent ouvrage, de l'Ancien Testament dans sa vie, son opinion définitivement négative de l'apostolat protestant en Chine, sa connaissance de la littérature mondiale et son attrait pour la poésie se retrouvent d'un article à l'autre. Ce qu'il faut en retenir est la fréquente influence de thèmes et de morales bibliques dans une littérature chinoise qui n'était pas, a priori, supposée s'intéresser à de tels sujets, ainsi dans l'œuvre de Mao Dun, pour prendre un des auteurs mondialement connu dont traite M. Gálik. Dans son introduction (p. 9-21), une spécialiste de la bible en Chine, Irene Eber, dessine l'orientation générale de l'ouvrage. On regrettera l'absence d'une bibliographie de l'auteur lui-même, afin de mieux percevoir ici la part des rééditions.

Lorsque le P. Malek a conduit une étape plus loin ses interrogations sur la place de la bible et du christianisme en Chine, en choisissant pour thème d'un travail collectif un titre aussi restrictif que The Chinese Face of Jesus Christ, il est arrivé qu'on juge alors sa démarche peu gratifiante. Mais il avait raison et le résultat est intéressant et varié. Avec trois gros volumes passionnants, une partie seulement du chemin vers une découverte nouvelle du message chrétien a été parcourue, et l'on attend la suite. Chaque volume comporte une douzaine d'articles de fond rangés par ordre chronologique, en anglais et parfois en allemand (pour certains des rééditions, surtout dans le premier volume), qui évaluent ce que la Chine a trouvé dans le Christ à chaque étape de son contact avec lui, puis une anthologie d'une centaine de pages destinée principalement aux non sinologues.

Le Volume 1 s'ouvre par une longue introduction richement documentée du maitre d'œuvre de l'ouvrage, qui justifie son choix du terme "visage " pour rechercher dans quelle mesure et sous quelle forme la figure christique a pu être transmise à la Chine. Et un avocat du diable lui répond, le dominicain Paul $\mathrm{H}$. Welte, qui a enseigné dans les grands séminaires chinois et qui met ici en doute les parallélismes usuels entre croyances et rites du taoïsme ou du bouddhisme d'une part, et du christianisme d'autre part, ou encore des assertions superficielles d'inculturation. Une première section de quatre contributions pose 
cependant le thème christique dans son contexte taoïste (Livia Kohn - je ne citerai dans les lignes qui suivent que quelques noms d'auteurs, parmi ceux qui donnent ici un article original -) et bouddhiste et évoque les diverses manières dont le nom de Jésus a été rendu selon l'allégeance ecclésiale des auteurs (J.O. Zetzsche). La seconde section est historique et concerne le nestorianisme (jingjiao) et le manichéisme (monijiao) de l'époque Tang ainsi qu'une stèle chrétienne de l'époque mongole (de 1344). L'anthologie finale de textes nestoriens et manichéens est tirée de l'édition réalisée en 1937 par le Japonais Saeki. En outre, un poème du célèbre Li Bai (ou Li Bo, du VIII ${ }^{e}$ siècle) est commenté comme étant une allusion au nestorianisme par un historien chinois, Wu Qiming (dont le propos est résumé par un remarquable connaisseur du christianisme jésuite entre Ming et Qing, Adrian Dudink, p. 385-391).

Le Volume 2 couvre la grande époque missionnaire des jésuites du $\mathrm{XVI}^{\mathrm{e}}$ au $\mathrm{XVIII}^{\mathrm{e}}$ siècle et les effets de l'évangélisme protestant au XIX ${ }^{\mathrm{e}}$ siècle. La représentation christique y occupe la plupart des contributions qui, phénomène rare dans un ouvrage collectif, restent assez bien concentrées sur le thème fédérateur. Pour les catholiques, sont exposés l'iconographie (Gauvin A. Bailey, Sun Yuming), l'enseignement occidental (celui d'Aleni par Gianni Criveller, ceux des figuristes par Claudia von Collani, des premiers dominicains par Miguel Ángel San Roman) et l'interprétation qu'en ont faite les convertis chinois (chrétiens confucéens par Paul Rule, Yang Tingyun à ses débuts par Whalen Lai, Zhu Zhongyuan par Dominic Sachsenmaier - ici en anglais, alors que son livre sur ce même personnage est en allemand). Du côté protestant, nous avons les diverses visions du Christ transmises par des missionnaires anglo-saxons et allemands (Ralph R. Covell) et les perceptions qu'en ont eues les Chinois au XIX ${ }^{\mathrm{e}}$ siècle (Jessie G. Lutz), les racines évangéliques du mouvement des Taiping (P. Richard Bohr), les visions populaires du Christ dans Chine impériale, notamment celles qui le tournent en un objet de mépris et de dérision (Lauren F. Pfister), des associations volontaires placées sous le nom de Jésus dans un milieu presbytérien du Henan (Margo S. Gewurtz). Bien que ne touchant pas au Christ en particulier mais à la christianisation en général, une contribution est à distinguer tout particulièrement : celle du mandchouisant italien Giovanni Stary, "Jesus introduced to the Manchus » (p. 599-611). Dans la ligne d'une intéressante réévaluation de la «mandchouité » de la dernière dynastie, les Qing, menée depuis les années 1990 dans les études sinologiques, est ici soulignée la difficulté supplémentaire que les jésuites ont rencontrée dans leurs tentatives d'apostolat en milieu mandchou au XVIII siècle : l'importance du chamanisme dans l'affirmation ethnique et culturelle des Mandchous. La partie anthologique est riche de traductions de morceaux écrits en chinois par les jésuites du XVII ${ }^{\mathrm{e}}$ siècle et par les Chinois eux-mêmes, convertis ou détracteurs au XVII ${ }^{\mathrm{e}}$ autant qu'au XIX ${ }^{\mathrm{e}}$ siècle.

L'époque moderne ayant fourni à l'éditeur une matière trop abondante, il l'a fragmentée en deux volumes. Le présent Volume $3 a$ concerne uniquement l'époque 
de la République (1912-1949), la seconde moitié du $\mathrm{xx}^{\mathrm{e}}$ siècle étant réservée pour le Volume 3b. La vision islamique de Jésus, qui ouvre le recueil, est mise en lumière par de très utiles traductions de citations tirées de trois ouvrages marquants de l'islam chinois : l'un des alentours de 1724 dû à Liu Zhi, le plus célèbres de 'âlim chinois, un autre de Lan Zixi en 1861, enfin un de Li Tingxian en 1919 (p. 847-867, par le grand maître de l'islamologie chinoise, Donald D. Leslie et son collaborateur habituel, Yang Daye). Les visions qui se succèdent ensuite sont essentiellement protestantes : Jésus au centre de l'apostolat protestant destiné aux sino-musulmans (F. Aubin); Jésus dans les écrits des théologiens protestants, l'un occidental (Karl Ludwig Reichelt, par Ekman P.C. Tam), les autres Chinois (Jésus Fils de Dieu pour Wang Mingdao, par Poling J. Sun ; un Jésus réformateur social pour Wu Leichuan, par le P. Malek lui-même ; un Jésus pur confucéen pour T.C. Chao/Zhao Zichen, par Winfried Glüer); et dans la littérature chinoise de l'époque républicaine sous l'influence de la bible des protestants, chez Lu Xun (Lewis S. Robinson, Anna Bujati), Mao Dun (Liang Gong, A. Bujati), Lin Yutang (Gotelind Müller) et même chez un jeune auteur japonais Akutagawa Ryûnosuke, qui livre un message ambigu dans une nouvelle ayant Shanghaï pour cadre (Francis K.H. So). Du côté catholique, on trouve le témoignage d'un homme politique converti au catholicisme (John C.H. Wu, par Matthias Christian) et l'apport littéraire et pictographique du périodique catholique de Hong Kong, dans les années qui ont suivi sa fondation en 1928 (Anthony S.K. Lam). Les vingt-quatre extraits de textes originaux, qui constituent l'anthologie complémentaire, laissent la parole aux auteurs autochtones cités dans les articles précédents et à quelques autres, Chiang Kai-shek notamment (p. 1308-1311).

Les volumes suivants vont conduire l'enquête jusqu'au XXI ${ }^{\mathrm{e}}$ siècle et apporteront la bibliographie générale et l'index avec glossaire chinois qui manquent jusqu'à présent, ainsi qu'une iconographie complémentaire (celle qui est incluse dans chaque volume étant déjà riche par elle-même). Il est un détail que le lecteur aimerait voir éclairci : quelle logique gère la répartition des contributions à l'intérieur de chaque volume alors qu'elle n'est pas alphabétique et ne semble guère thématique ? Ce soupçon de critique mis à part, on ne peut qu'admirer l'ampleur de l'entreprise conduite par le P. Malek, dans laquelle autant le spécialiste de l'histoire des religions ou des missions peut puiser que le comparatiste en littérature ou l'historien de la Chine. 